\title{
Some new approaches to the study of the Earth's magnetic field reversals
}

\author{
Paola De Michelis $\left({ }^{1}\right)$ and Giuseppe Consolini $\left({ }^{2}\right)$ \\ (1) Istituto Nazionale di Geofisica e Vulcanologia, Roma, Italy \\ ( $\left.^{2}\right)$ Istituto di Fisica dello Spazio Interplanetario, CNR, Roma, Italy
}

\begin{abstract}
Paleomagnetic studies clearly show that the polarity of the magnetic field has been subject to reversals. It is generally assumed that polarity intervals are exponentially distributed. Here, the geomagnetic polarity reversal record, for the past $166 \mathrm{Ma}$, is analysed and a new approach to the study of the reversals is presented. In detail, the occurrence of $1 / f$-noise in the Power Spectral Density (PSD), relative to geomagnetic field reversals, the existence of a Zipf's law behaviour for the cumulative distribution of polarity intervals, and the occurrence of punctuated equilibrium, as shown by a sort of Devil's staircase for the reversal time series, are investigated. Our results give a preliminary picture of the dynamical state of the geomagnetic dynamo suggesting that the geodynamo works in a marginally stable out-of-equilibrium configuration, and that polarity reversals are equivalent to a sort of phase transition between two metastable states.
\end{abstract}

Key words Earth's magnetic field - geomagnetic reversals - time series analysis

\section{Introduction}

At the beginning of the last century, the French scientists P. David and B. Brunhes made the earliest demonstration that the geomagnetic field has changed its polarity in the past. The idea that geomagnetic polarity could change was controversial, and for many years sceptics sought alternative interpretations. The hypothesis became widely accepted only in the early 60 s and we can say that this is probably the major phenomenological discovery of the twentieth century in the field of paleomagnetism. However, geomagnetic polarity reversal remains one of nature's most enigmatic phenomena. From a

Mailing address: Dr. Paola De Michelis, Istituto Nazionale di Geofisica e Vulcanologia, Via di Vigna Murata 605, 00143 Roma, Italy; e-mail: demichelis@ingv.it theoretical point of view the possibility that the geomagnetic field could change its polarity was well known. Indeed, the magnetohydrodynamic description of the geodynamo is insensitive to the sign of the magnetic field: i.e. the induction equation is invariant under magnetic field parity transformation $(\boldsymbol{B} \rightarrow-\boldsymbol{B})$. Therefore, the geodynamo can have a polarity opposite to the directly observable normal polarity as an equally viable solution. If a mechanism for transition between these two states exists, we should expect normal and reversed polarity states with equal probability. From dynamo theory we can conclude that the geodynamo appears to operate in two regimes, one in which reversals occur and one in which they do not. Although the conditions that give rise to these two regimes are not completely understood, it is known that the reversal sequence of the Earth's magnetic field contains information about Earth's core processes. In particular, from the analysis of the length distribution of the polarity intervals it is possible to try to model the reversal mechanism while from the analysis of the rate of occurrence 
of these reversals it is possible to try to gain information on gradually changing conditions at the core-mantle boundary. Indeed, it is generally accepted that changes in the frequency of field reversals reflect the dynamic evolution of the fluid core (Jones, 1977; McFadden and Merrill, 1984) and that the analysis of long-term trends in geomagnetic reversal frequency might provide important information on core-mantle coupling (e.g., McFadden and Merrill, 1984, 1986; Courtillot and Besse, 1987; Gubbins, 1987; Marzocchi et al., 1992). Thus it is important to analyse the reversal sequence and to extract all the available information.

Modern reversal chronologies show more than 300 successful reversals in the past 166 Ma. Common estimates for the duration of a polarity transition are around a few thousand years (1000-8000 years). However, remarkably different values for the duration of a magnetic field reversal are often advocated even if there are reasons to think that these values are artefacts. In geological terms the duration of a polarity transition is a very short time and is short relative to the typical length of polarity interval. So, in first approximation, it is possible to think of a reversal as a rapid, almost instantaneous event. This then naturally leads to the concept that the reversal process is a renewal process. This idea was originally suggested by Cox (1968) who was the first to notice the irregular lengths of stable polarity intervals and to suggest that one might model reversals as a renewal process triggered by instabilities in fluid motions in Earth's outer core. In the hypothesis in which the reversals are generated by a renewal process, the duration of polarity intervals must be independent and consequently the reversal process must be a Poisson process, i.e. a process in which the probability of a future event does not depend on the time since the last event. Under the Poisson model, one expects that although the lengths of individual stable polarity intervals are highly variable, there is a characteristic rate of reversal if the process is averaged over a sufficient time interval. However, the observed distribution of reversals exhibits two departures from the basic Poisson model. Firstly, there is a paucity of short intervals in the magnetostratigraphic records, which could be a consequence both of our inability to detect very short intervals and an inability of the core to generate new reversals during some short time interval immediately following a transition (McFadden and Merrill, 1993). In the second place, the occurrence of geomagnetic reversals appears to be time-dependent. The average rate at which reversals occur has varied significantly over time scales on the order of $10^{8}$ years. In particular, the reversal rate appears to have decreased from just over 3 per million years at about $160 \mathrm{Ma}$ ago down to zero at about $118 \mathrm{Ma}$ ago and then increased from zero at about $83 \mathrm{Ma}$ ago to around 5 per million years in recent times (McFadden and Merrill, 1997). This could mean that the geomagnetic reversal process is a non-stationary process at least over such time scales. It is important to emphasize that only the existence of this nonstationarity, not its details, has been well established. Indeed, the origin of the nonstationarity in reversal rate has been interpreted in different ways in the past decade. The nonstationarity could be, for example, a manifestation of deterministic chaos within the geodynamo or a consequence of gradual changes in the core mantle boundary conditions. Recently, longer-term changes in reversal rates have been linked to plume activity at the core-mantle boundary (Courtillot and Besse, 1987; Loper, 1992) or to the arrival at the core mantle boundary of cold material from a mantle event (Gallet and Hulot, 1997) or to changes at the inner-core/outer-core boundary (Kent and Smethurst, 1998). Nowadays the favoured conjecture is that the change in reversal rate reflects the evolution in spatial variability of some parameter, such as heat flux, at the CoreMantle Boundary (CMB) (Merill et al., 1996; Glatzmaier et al., 1999).

Taking into account that reversal sequences are not stationary and that a reversal requires a finite amount of time, one can think that there has to be some statistical correlation between the lengths of successive polarity intervals. In this case, the process cannot be truly Poisson and it has been shown (Naidu, 1971; Phillips, 1977) that a gamma distribution could provide a better fit to the observed intervals. It has also been suggested (McFadden and Merrill, 1984; Lutz and Watson, 1988) that the process of geomagnetic reversals may be consistent with a 
nonstationary Poisson process, which is a special case of gamma process where there is no memory of how long it has been since the previous event. We remark that a gamma distribution generally occurs in out-of-equilibrium dynamical systems (Sornette, 2000). It has also been found (Gaffin, 1989, Seki and Ito, 1993) that the cumulative distribution of the polarity intervals follows a power law. In particular, Seki and Ito (1993) interpreted their results as indicating that the geodynamo is marginally stable and that the geomagnetic polarity reversal is a kind of critical phenomenon. In particular they presented a simple model (a dissipative version of the Q2R Ising model) in which the geodynamo was assumed to be a system of magnetic spins in a critical phase-transition state. By numerical simulation, they found that intervals of polarity reversals in the model follow a power law at a critical state. They also improved their model (Seki and Ito, 1999) to make it more realistic and to obtain a power exponent closer to the observed value. The revised model is a coupled map lattice where the elements themselves evolve and reverse polarity autonomously according to the Lorentz map obtained from Rikitake dynamo dynamics. Analysing the behaviour of the system and the distribution of polarity reversals for various values of parameters of the model they suggested that the dynamo in the Earth's core is in a chaotic turbulent state. Similar conclusions were arrived at by Consolini et al. (2000) by investigating the multifractal features of the polarity interval time series.

In this paper, an attempt is made to study the geomagnetic reversal record from the viewpoint of dynamical complex systems theory. Reversals of the Earth's dipole field are in fact associated with magnetohydrodynamic processes in the convecting outer core and for this reason it is important to apply any new ideas which can give additional insight into this fundamental aspect of planetary magnetism. Moreover, there is general agreement that the dynamo equations are sufficiently nonlinear to allow reversing solutions and that simple analogue equations, such as those of the coupled disk dynamo of Rikitake, exhibit spontaneous and apparently random polarity reversals. Consequently one possible answer to the geomagnetic field time reversals debate is to describe these events as typical nonlinear behaviour of a highly complex dynamical system especially if one takes into account that nonlinear systems are capable of a vast range of responses. In this picture we investigated the possible occurrence of Zipf's law, 1/f noise, and punctuated-equilibrium in the geomagnetic polarity reversals, analysing all reversal data back to about $166 \mathrm{Ma}$ ago.

\section{The rank-ordering statistics and the Zipf's law}

In the last three decades, growing interest has been devoted to many natural phenomena that are characterised by Power-Law Distributions (PLDs). Although the asymptotic existence of power-laws is a well-established fact in statistical physics and critical phenomena, powerlaw behaviours are also considered to be one of the most striking features of complex systems, i.e. of those systems, which consist of several interacting subunits, and whose phenomenological laws, describing the global behaviour, cannot be surmised from the elemental laws that regulate the evolution of its elementary parts. Power-law distributions are commonly taken as a signature of an underlying self-similarity. As a matter of fact, in contrast to exponential distributions, a PLD indicates the absence of a characteristic size: i.e. there is no upper limit on the event size. We remark that in the case of a phenomenon whose events follow a PLD, the largest events completely dominate the physical processes (see for example what happens in the case of earthquakes).

From a practical point of view, a PLD is represented by a linear trend in a log-log plot of the frequency or cumulative number as a function of size. One of the main and more debated questions on PLD is the validity of such a distributions for very large events, which are generally undersampled. One way to solve such a problem when only a limited statistics of largest events is available is to use the rank-ordering technique (Sornette et al., 1996; Sornette, 2000). George Kingsley Zipf of Harvard University initially introduced this technique in his book (Zipf, 1949), where he made many striking 
observations of simple regularities in human systems. The most known regularity observed by Zipf was the distribution of how often a given word appears in a piece of literature. In any case, rank-ordering statistics was subsequently applied to a wide range of research fields.

Given a set of numbers $\{n\}$ upper limited, the rank-ordering technique consists in evaluating how many (rank order $r$ ) of these numbers are smaller than a certain cut-off value $n^{*}$, and then in plotting $n^{*}$ as a function of $r$. Thus Zipf's plot is a log-log plot of the cumulative distribution with an interchange of axes $\left[n^{*}=f(r)\right.$ instead of $r=f\left(n^{*}\right)$ ]. Although the statistical analysis of the cumulative distribution and of the rankordering statistics seems to be equivalent, they are significantly different, the analysis being completely determined by the uncertainties of the quantity plotted in the ordinates. In other words, the rank-ordering statistics provide a different emphasis on the extreme and rare events.

The existence of a power law behaviour $\left(n^{*} \approx r^{-\alpha}\right)$ in the Zipf's plot is the indication of a certain amount of correlation and selforganisation. The original Zipf's law for words is characterised by a scaling exponent $\alpha \approx 1.0$. Anyway, different values of $\alpha$ may be recovered in different physical systems meaning a different degree of correlation and self-organisation. For example in the case of DNA sequences it was found $\alpha \approx 0.3$ (Mantegna et al., 1994). Other examples of the Zipf's laws come from rankordering statistics of stock-market variations, of city distributions (Marsili and Zhang, 1998), of large earthquakes (Sornette et al., 1996), etc.

Here, we will make use of rank-ordering statistics to investigate the existence of Zipf's law in the geomagnetic chron distribution.

\section{Data description and analysis}

The geomagnetic polarity time scale compiled by Cande and Kent $(1992,1995)$ represents a great improvement in defining the chronology of reversals from the Late Cretaceous to the present. Because the time scale of Cande and Kent (1995) does not go back further than $83 \mathrm{Ma}$, we have merged it with the portion of the scale for the time interval 83-166 Ma (Ogg, 1995) to investigate the statistical properties of reversals from the present to $166 \mathrm{Ma}$ ago. The whole sequence contains 332 intervals of constant polarity, 144 intervals of constant polarity before the Cretaceous superchron and 188 afterwards.

The sequence of polarity intervals according to the time scale of Cande and Kent $(1992,1995)$ and Ogg (1995) is presented in fig. 1, where the polarity interval lengths $\Delta T(x)$ are plotted as a function of the order of occurrence $(x)$. Ages (dates) of geomagnetic inversion that indicate the borders of intervals with different main

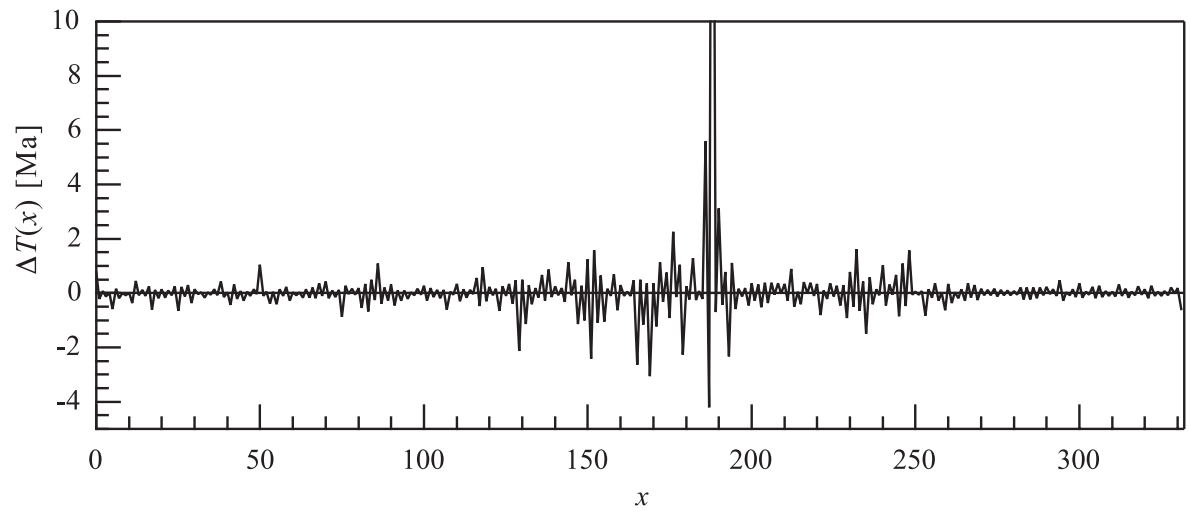

Fig. 1. The time series of geomagnetic polarity interval duration $(\Delta T(x))$ for both polarities as a function of order of occurrence $(x)$. The $y$-axis was limited to $10 \mathrm{Ma}$. 
geomagnetic field polarity are presented in this scale with an accuracy of $0.001 \mathrm{Ma}$. This value is of the same order of magnitude as the process of polarity change, which is around 3.0-3.5 ka. In this analysis we have not made any distinction between excursions, subchrons, chrons, and superchrons. All these events can be considered manifestations of the dynamics of the Earth's dynamo. Also, due to the inherent uncertainties in the determination of the reversal ages, we have set the average conventional scale resolution to be about $0.01 \mathrm{Ma}$, even though the accuracy of determination of individual events may be one order higher. Although we know that the uncertainties which remain in the precise absolute dates of the geomagnetic polarity time scale make their detailed analysis delicate, we believe that they would not notably modify the broad description we intend to present hereafter.

As already noted, there is a clear nonstationarity in this record, which becomes evident when the distribution of the geomagnetic reversal rate on a $5 \mathrm{Ma}$ time scale is analysed (McFadden and Merrill, 1984). The obtained trend in fact suggests that the reversal rate has gradually increased from the end of the Cretaceous superchron to the present and this is evidence of the nonstationary behavior of the geomagnetic polarity time scale. However, we must take into account that this interpretation, even though it is compatible with the data, is strongly guided by the way the data is presented and by the choice of the strong smoothing made on the series (as also noted by Gallet and Hulot, 1997). The nonstationarity of the reversal process could cause some difficulties in defining the statistical properties of the record. Gaffin (1989) and Marzocchi (1997) have suggested that some results obtained in previous papers could be artefacts of the non-stationarities present in the series. Nevertheless, we believe that the problem of the nonstationarity of the reversal process is not relevant for our analysis.

First of all, in order to study the nature of the polarity reversals we analysed the Power Spectral Density (PSD), and investigated the occurrence of scaling laws (Zipf's law) in the cumulative distribution of the chrons. To evaluate the PSD of the chron time series we generated a surrogate two-state $( \pm 1)$ time series covering the entire period length of $166 \mathrm{Ma}$ with a resolution of 0.01 Ma. The state +1 was associated with normal polarity and -1 with the reversed polarity.

Figure 2 shows the obtained Power Spectral Density (that is, the squared magnitude of the Fourier transform) for the analysed polarity reversal time series. Two different scaling regions and a spectral break can be identified. Indeed the Power Spectral Density shows a $1 / f$ spectrum for times longer than $T_{0}=1 / f_{0} \approx 0.4 \mathrm{Ma}$, while for time scales shorter than $T_{0}$ it scales as $1 / f^{2}$, which is reminiscent of a stochastic motion. The physical meaning of the $1 / f^{2}$ region should be limited to the random nature of the polarity sequence (i.e. of the random distribution of the $\pm 1 \rightarrow \Upsilon 1$ transition) in the surrogate time series. The occurrence of a $1 / f$ spectrum on longer time scales is evidence of a certain amount of longtime correlation in the sequence of polarity reversals, and of a complex time evolution of the geodynamo. We stress that $1 / f$-noise spectra represent one of the most relevant features of out-of-equilibrium and complex systems whose evolution is characterised by first-order transitions between metastable configurations (Bak, 1996).

Because $f_{0}$ (which represents the value of the spectral break) is the characteristic frequency for a sinusoidal signal, we must conclude that the typical time interval between two polarity reversals is

$$
\delta \tau_{0}=1 / 2 f_{0}=T_{0} / 2 \approx 0.22 \mathrm{Ma} .
$$

This value is in good agreement with the mean length of a polarity interval given in the literature which is about $0.3 \mathrm{Ma}$ even though its value depends on the number of short events present in the magnetic polarity time-scale. Lowrie and Kent (1983) have in fact discussed the effect of adding short events to the magnetic polarity timescale and they have shown that this has a dramatic effect on the polarity intervals distribution and on the mean length of a polarity interval, which is reduced from $0.314 \mathrm{Ma}$ to $0.175 \mathrm{Ma}$.

In order to study the possible occurrence of Zipf's law for the cumulative distribution of chron duration, we denote by $q_{\delta \tau}$ the number of 


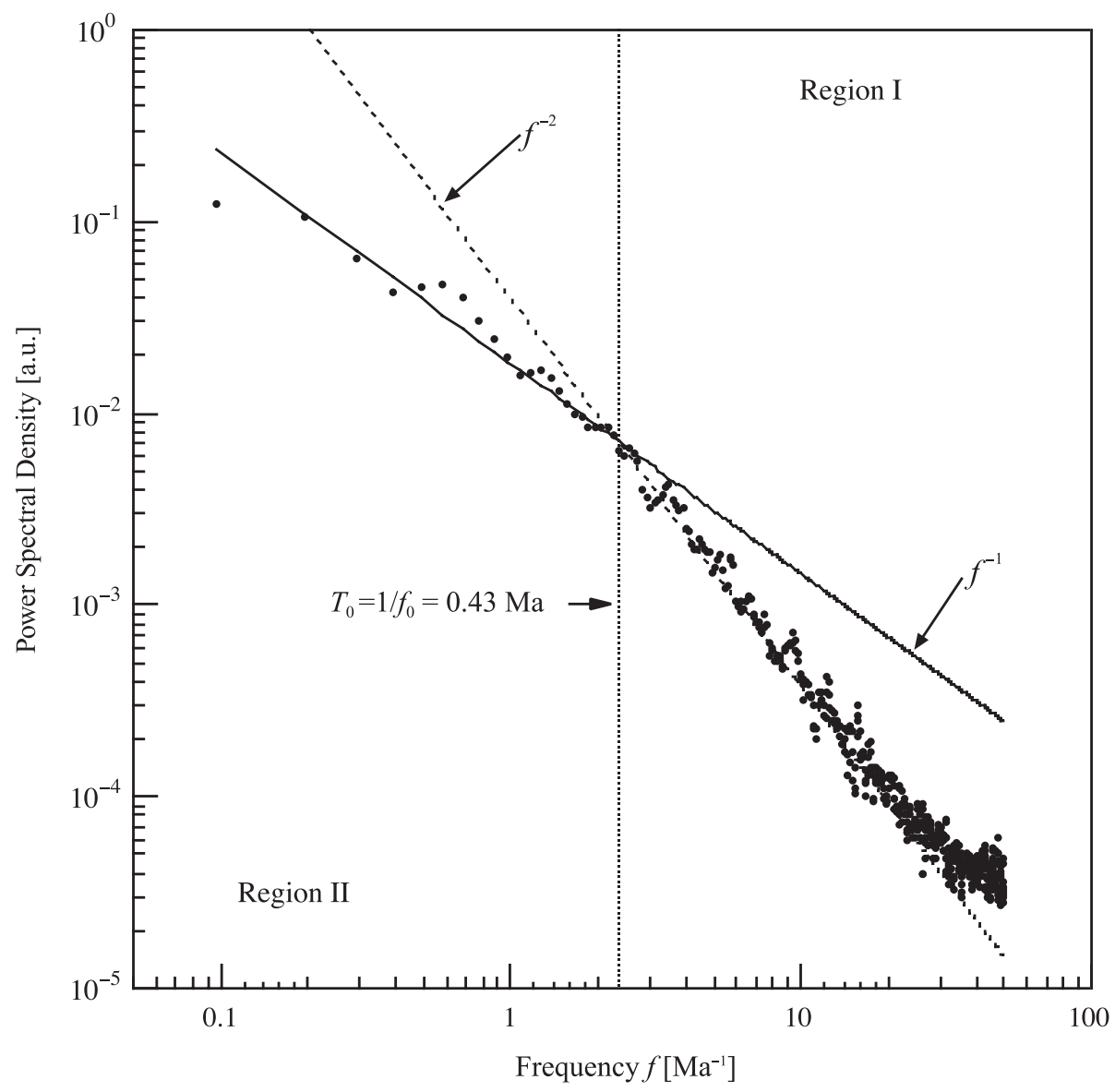

Fig. 2. The PSD, in arbitrary units (a.u.), of the surrogate polarity time series. The solid and dashed lines are nonlinear power-law best fits. The dotted vertical line indicates the position of the spectral break.

polarity intervals having a length equal to $\delta \tau$, and we define the rank $r$ as

$$
r(\delta \tau)=\int_{\delta \tau}^{\infty} q_{\delta \tau^{\prime}} d \delta \tau^{\prime} .
$$

This means that the rank $r(\delta \tau)$ is the number of polarity intervals which last longer than $\delta \tau$.

Figure 3 shows the rank order statistics of the polarity reversal time intervals obtained. A power-law $\delta \tau=A_{0} r^{-\alpha}$ applies for ranks $r \leq 100$ with a characteristic scaling exponent $\alpha$ of $\alpha \approx$ $\approx(0.76 \pm 0.01)$. This indicates that the polarity reversal dynamics obeys Zipf's law behaviour for $r \leq 100$. The existence of a Zipf's law means that for time scales longer than 0.25 Ma the geomagnetic polarity reversal phenomenon exhibits scale invariance and/or self-similar features, and that a certain amount of selforganization must occur in the geodynamo evolution. In terms of the distribution of polarity chron durations Zipf's law is equivalent to a power law distribution. As a matter of fact our result is in good agreement with the analyses of Seki and Ito $(1993,1999)$ and Gaffin (1989), which found a power law distribution with a 


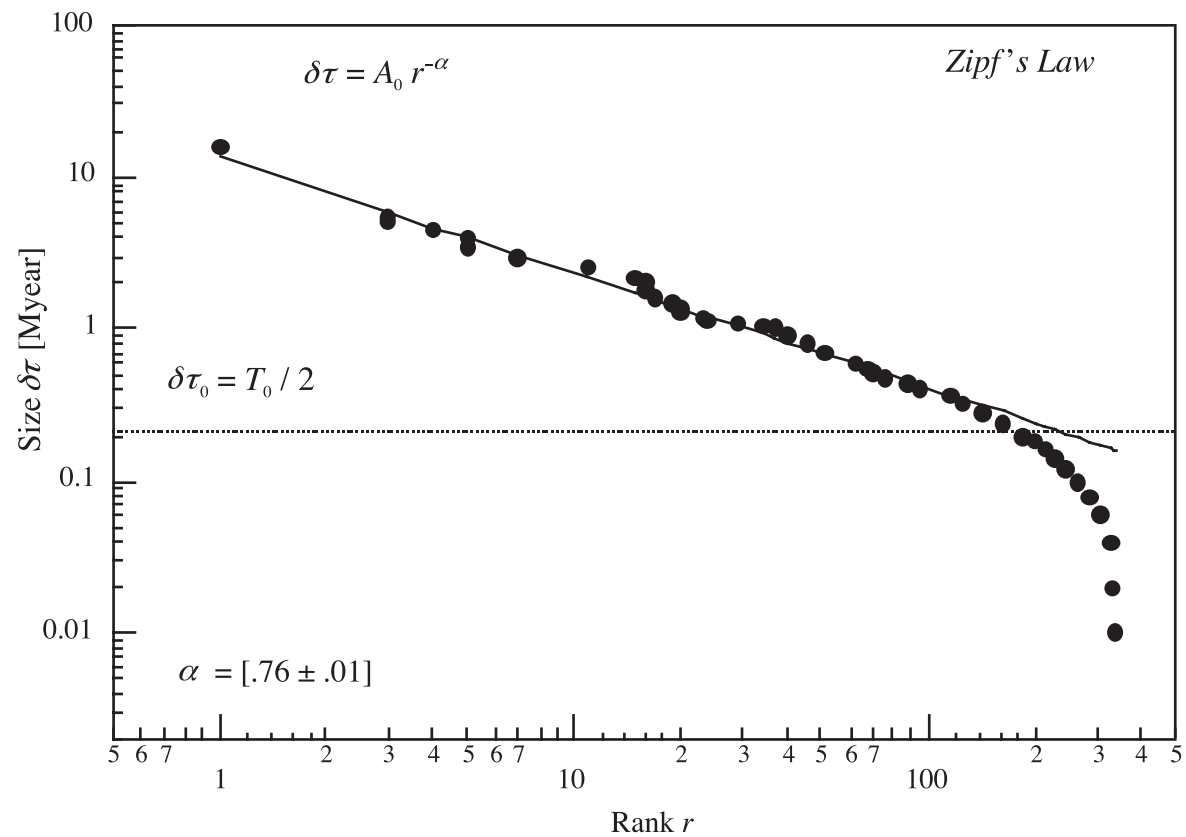

Fig. 3. The ranking distribution relative to the polarity time intervals. The solid line is a nonlinear power-law best fit. The horizontal dotted line indicates the characteristic time-scale evaluated on the basis of the PSD (see text).

scaling exponent near - 1.5 for chron durations longer than 0.23 Ma. Moreover, the existence of such a power-law statistics could be related to the occurrence of «punctuated equilibrium».

The concept of punctuated equilibrium was introduced by Gould and Eldredge (1993) in the field of evolution in natural history, and refers to a system which is characterized by periods of relative quiescence, interrupted abruptly by rapid change (the crisis). This phenomenon may be taken as an indication of metastability in the dynamics of a system. Punctuated equilibrium has been shown to be a common feature of several complex systems (Bak, 1996). In terms of polarity chron duration punctuated equilibrium could be paraphrased as: «the existence of periods of stable polarity punctuated by crises where a polarity inversion occurs». This phenomenon may be visualized by the existence of a Devil's staircase for the accumulated activity.

The Devil's staircase was introduced by G. Cantor in the 19th century as a special mathe- matical object generated by a multiplicative process and characterized by self-similarity. The name Devil's staircase comes from the existence of very large and very small steps alternating in a random way. Although for a long time it was thought that physical and real systems cannot share the mathematical features of the Devil's staircase, in the last 3 decades it has been clearly demonstrated that several complex system are able to show behaviours that may be represented in terms of a Devil's staircase (Bak and Bruisma, 1982; Jensen et al., 1983; Bak, 1996).

In order to check the occurrence of punctuated equilibrium for geomagnetic reversals we introduced a surrogate «Accumulated Activity» $A A(t)$ as

$$
A A(t)=\int_{0}^{t}\left|\partial_{t^{\prime}} x\left(t^{\prime}\right)\right| d t^{\prime}
$$

where $x\left(t^{\prime}\right)$ is the binary time series of polarity 


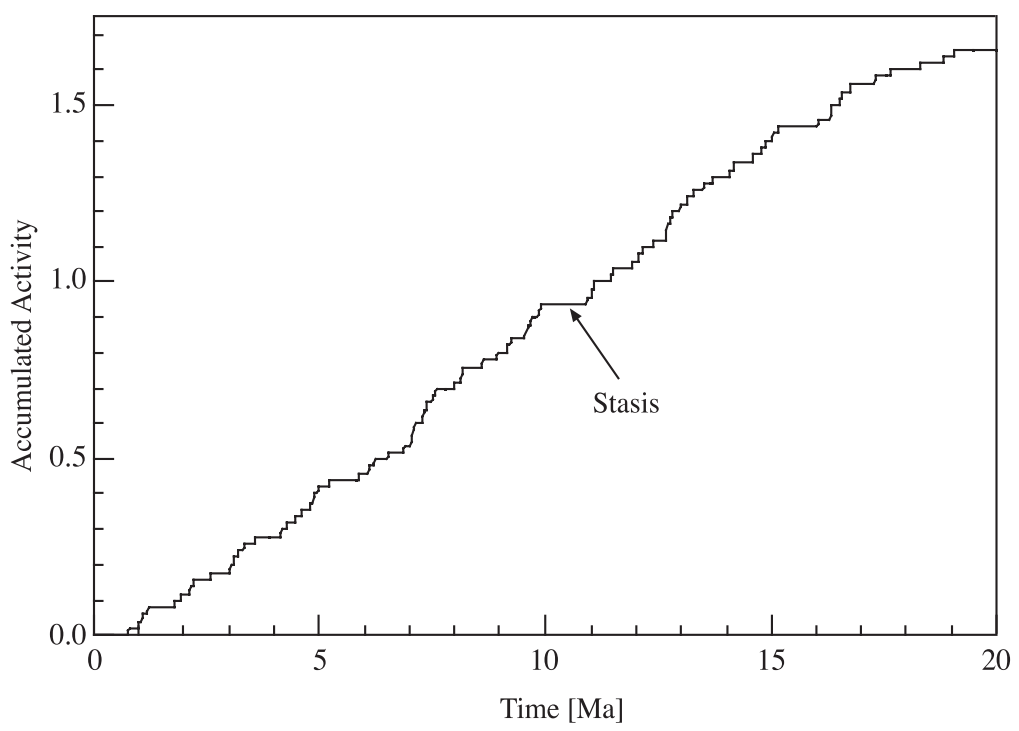

Fig. 4. The Devil's staircase for the geomagnetic polarity time series resulting from the Accumulated Activity $A A(t)$.

reversals. Figure 4 shows the result obtained for the Accumulated Activity. The plot of the defined Accumulated Activity looks very similar to a Devil's staircase. Plateaus of stasis (i.e. the steps of the staircase) do not follow one another in a regular way. This result which is a signature of the occurrence of punctuated equilibrium, may be read as an indication of metastability and can be linked to a complex configurationally space for the geodynamo. Moreover, the irregular character of the stasis sequence confirms our previous findings of a multifractal and intermittent character of the geomagnetic polarity reversal series (Consolini et al., 2000).

\section{Summary and conclusions}

In this paper, we have presented a study of the geomagnetic chron time length sequence to infer information on the dynamical state of the geomagnetic dynamo.

In order to do this we performed power spectral analysis of polarity reversal time series using the generally accepted geomagnetic polarity time scale compiled by Cande and Kent
(1992, 1995) and Ogg (1995) which covers the last $166 \mathrm{Ma}$. We found that the power spectrum shows a $1 / f$ spectrum, where $f$ is the frequency, for times longer than $T_{0} \approx 0.5 \mathrm{Ma}$ while for times shorter than $T_{0}$ it scales as $1 / f^{2}$. The most striking feature of this result is the existence of a crossover point that divides the total time interval into two different parts with different scaling properties. We also evaluated the possible occurrence of Zipf's law for the cumulative distribution of chron duration and we have found that the polarity reversal dynamics obeys Zipf's law for time scales longer than $0.25 \mathrm{Ma}$. This result permitted us to conclude that the geomagnetic polarity reversal phenomenon exhibits scale invariance and/or self-similar features. Finally, we investigated the possibility that the phenomenology of the polarity reversals in the geomagnetic dynamo is that of punctuated equilibrium. We evaluated the accumulated activity of the polarity reversal time series and obtained a trend quite similar to a Devil's staircase with plateaus of stasis (distributed according to a power law) punctuated by short periods of rapid activity.

These results give a preliminary picture of the dynamical state of the geomagnetic dynamo. 
The fact that the geomagnetic dynamo is characterized by punctuated equilibrium means that the geodynamo dynamics is characterized by metastability. Combining this result with the observed scale invariance in the polarity interval distribution, we can consequently assume that the geomagnetic dynamo might be in a marginally stable out-of-equilibrium configuration and that the polarity reversals are equivalent to a sort of phase transition between two metastable states. We suggest that the dynamics of the geomagnetic dynamo could be equivalent to that of a stochastic system near criticality and consequently that the geomagnetic dynamo evolves as a system near a dynamic critical point. We believe that our findings strongly support previous work by ourselves (Consolini et al., 2000) and by Seki and Ito $(1993,1999)$.

We would like to remark that this hypothesis has been also suggested by McFadden and Merrill (2000) who considered the existence of a mechanism for punctuated equilibrium within the core. In particular, they suggested that, for example, phenomena such as the sudden breakup of a stratified layer in the outermost core could lead to a rapid change in boundary conditions that could be responsible for the punctuated evolution manifested in the sudden jumps in the reversal rate. Here, reversals would be equivalent to noise-induced cooperative phase transitions. This last picture fits well with our latest result of the possible occurrence of stochastic resonance in geomagnetic polarity reversals (Consolini and De Michelis, 2003).

The possible relevance of our findings to a possible critical dynamical state of the geodynamo calls for further investigation, particularly from a theoretical point of view.

\section{REFERENCES}

BAK, P. (1996): How Nature Works: the Science of SelfOrganized Criticality (Copernicus) (Springer-Verlag, New York, NY, U.S.A.), pp. 336.

BAK, P. and R. BRUINSMA (1982): One-dimensional Ising model and the complete Devil's staircase, Phys. Rev. Lett., 49, 249-251

CAnDE, S.C. and D.V. KenT (1992): A new geomagnetic polarity time scale for the Late Cretaceous and Cenozoic, J. Geophys. Res., 97, 13,917-13,951.
CANDE, S.C. and D.V. KENT (1995): Revised calibration of the geomagnetic polarity timescale for the Late Cretaceous and Cenozoic, J. Geophys. Res., 100, 6093-6095.

Cox, A. (1968): Lengths of geomagnetic polarity intervals, J. Geophys. Res., 73, 3247-3260.

Courtillot, V. and J. Besse (1987): Magnetic field reversals, polar wander, and core-mantle coupling, Science, 237, 1140-1147.

Consolini, G. and P. De Michelis (2003): Stochastic resonance in geomagnetic polarity reversals, Phys. Rev. Lett., 90, 058501-4.

Consolini, G., P. De Michelis and A. Meloni (2000): Multifractality and punctuated equilibrium in the Earth's magnetic field polarity reversals, Gephys. Res. Lett., 27, 293-296.

GAFFIN, S. (1989): Analysis of scaling in the geomagnetic polarity reversal record, Phys. Earth Planet. Int., 57, 284-290.

Gallet, Y. and G. Hulot (1997); Stationary and nonstationary behaviour the geomagnetic polarity time scale, Geophys. Res. Lett., 24, 1875-1878.

GlatzMAIER, G.A., R.S. CoE, L. HONGRE and P.H. RoBERTS (1999): The role of the Earth's mantle in controlling the frequency of geomagnetic reversals, Nature, 401, $885-890$

Gould, S.J. and N. Eldredge (1993). Punctuated equilibrium comes of age, Nature, 366, 223-227.

GuBBINS, D. (1987): Mechanism for geomagnetic polarity reversals, Nature, 326, 167-169.

Kent, D.V. and M.A. Smethurst (1998): Shallow bias of paleomagnetic inclinations in the Paleozoic and Precambrian, Earth Planet. Sci. Lett., 160, 391-402.

Jensen, M.H., P. BAK and T. BoHR (1983): Complete Devil's staircase, fractal dimension and universality of mode-locking structure in circle map, Phys. Rev. Lett., 50, 1637-1639.

JONES, G.M. (1977): Thermal interaction of the core and mantle and long-term behaviour of the geomagnetic field, J. Geophys. Res., 82, 1703-1709.

LOPER, D. (1992): On the correlation between mantle plum flux and frequency of reversals of the geomagnetic field, Geophys. Res. Lett., 19, 25-28.

LOWRIE, W. and D.V. KENT (1983): Geomagnetic reversal frequency since the Late Cretaceous, Earth Planet. Sci. Lett., 62, 305-313.

LUTZ, T.M. and G.S. WATSON (1988): Effects of long-term variation on the frequency spectrum of the geomagnetic reversal record, Nature, 334, 240-242.

Mantegna, R.N., S.V. BuldyreV, A.L. Goldberger, S. Havlin, C.-K. Peng, M. Simons and H.E. Stanley (1994): Linguistic features of noncoding DNA sequences, Phys. Rev. Lett., 73, 3169-3172.

MARSILI, M. and Y.-C. ZHANG (1998): Interacting individuals leading to Zipf's law, Phys. Rev. Lett., 80, 2741-2744.

MARZOCCHI, W. (1997): Missing reversals in the geomagnetic polarity timescale: their influence on the analysis in constraining the process that generates geomagnetic reversals, J. Geophys. Res., Solid Earth, 102 (B3), 5157-5171.

Marzocchi, W., F. Murlagia and P. Paruolo (1992): The correlation of geomagnetic reversals and mean 
sea level in the last 150 m.y., Earth Planet. Sci. Lett., 111, 383-393.

MCFADDEN, P.L. and R.L. MERRILL (1984): Lower mantle convection and geomagnetism, J. Geophys. Res., 89, 3354-3362.

MCFAdDEN, P.L. and R.L. MERRILL (1986): Geodynamo energy source constraints from paleomagnetic data, Phys. Earth Planet. Int., 43, 22-33.

MCFADDEN, P.L. and R.L. MERRILL (1993): Inhibition and geomagnetic field reversals, J. Geophys. Res., 98, 6189-6199.

MCFADDEN, P.L. and R.L. MERRILL (1997): Asymmetry in the reversal rate before and after the Cretaceous normal polarity superchron, Earth Planet. Sci. Lett., 149, 43-47.

MCFADDEN, P.L. and R.L. MERRILL (2000): Evolution of the geomagnetic reversal rate since $160 \mathrm{Ma}$ : is the process continuous?, J. Geophys. Res., 105, 28,455-28,459.

MERRRILL, R.T., M.W. MCELHINNY andP.L. MCFADDEN (1996): Magnetic field of the Earth: paleomagnetism, the core and the deep mantle (Academic, San Diego), pp. 531.

NAIDU, P.S. (1971): Statistical structure of geomagnetic field reversals, J. Geophys. Res., 76, 2649.

OGG, J.G. (1995): Magnetic polarity time scale of the
Phanerozoic, in Global Earth Physics: a Handbook of Physical Constants, edited by T.J. AHRENS (Amer. Geophys. Union, Washington, DC), 240-270.

PHILLIPS, J.D. (1977): Time variation and asymmetry in the statistics of geomagnetic reversal sequences, J. Geophys. Res., 82, 835-843.

SeKI, M. and K. ITO (1993): A phase-transition model for geomagnetic polarity reversals, J. Geomagn. Geoelect., 45, 79-88.

SEKI, M. and K. ITO (1999): A coupled map lattice model for geomagnetic polarity reversals that exhibits realistic scaling, Earth Planet Space, 51, 395-402.

SORNETTE, D. (2000): Critical Phenomena in Natural Sciences. Chaos, Fractals, Self-organization and Disorder: Concepts and Tools (Springer-Verlag, Heidelberg), pp. 450.

SoRnetTe, D., L. KNOPOFF, Y.Y. KAGAN and C. VANNESTE (1996): Rank-ordering statistics of extreme events: application to the distribution of large earthquakes, $J$. Geophys. Res., 101, 13,883-13,893.

ZIPF, G.K. (1949): Human Behavior and the Principle of Least-Effort (Addison-Wesley, Reading, Mass).

(received January 8, 2003;

accepted June 20, 2003) 